\title{
CONOCIMIENTO PROFESIONAL DE LOS PROFESORES DE EDUCACIÓN FÍSICA COLOMBIANOS Y MEXICANOS, SOBRE ACTIVIDAD FÍSICA
}

\author{
PROFESSIONAL KNOWLEDGE OF COLOMBIAN AND MEXICAN PHYSICAL EDUCATION \\ TEACHERS, ABOUT PHYSICAL ACTIVITY
}

\author{
Dr. Alberto Calderón García, Ph.D $D^{1}$ \\ Lic. Ramiro Andrés Alzate Lubo ${ }^{2}$
}

\section{Resumen}

\begin{abstract}
La actividad física se ha convertido a través de los últimos años, en un movimiento que ha tomado fuerza en el campo conceptual y disciplinar de la educación física, trayendo como consecuencia su incorporación dentro de los programas de promoción de la salud y prevención dela enfermedad, a nivel latinoamericano y mundial con el fin de contribuir al mejoramiento de los estilos de vida de sus habitantes, mejorar sus condiciones de convivencia y, en términos generales, incrementar los niveles de calidad de vida de la población. En este sentido, este ejercicio investigativo nos muestra una pequeña mirada del conocimiento profesional que tienen los profesionales de la educación física y el deporte acerca de la actividad física en dos países latinoamericanos (México y Colombia) y de sus principales variables para su desarrollo de manera exitosa.
\end{abstract}

Como resultado es posible evidenciar algunas similitudes y diferencias que existen en torno a este conocimiento de contenido propio de la educación física a través de un estudio comparativo de pares que se desempeñan en los países mencionados, de esta manera contribuir a la construcción de un nuevo conocimiento unificado que permita la consolidación de un modelo pedagógico y didáctico propio de los maestros de la educación física y el deporte, enriqueciendo a su vez no sólo su conocimiento disciplinar sino también su Conocimiento Pedagógico General y el Conocimiento Didáctico del Contenido (CDC) factores fundamentales en la estructuración del conocimiento profesional del los profesores de educación física.

Palabras clave: actividad física, educación física, movimiento, salud, pedagogía, didáctica, conocimiento profesional, conocimiento pedagógico del contenido, conocimiento disciplinar o de contenido.

\section{Abstract}

The physical activity has turned across last years, in a movement that has taken force in the conceptual field and to discipline of the physical education, bringing as consequence his incorporation inside the programs of promotion of the health and prevention of disease, to Latin-American and world level in order to contribute to improvement of the ways of life of his inhabitants, to improve his conditions of conviviality and, in general terms, to increase the quality levels of life of the population. In this respect, this investigative exercise shows us a small look of professional knowledge that there have the professionals of the physical education and the sport about the physical activity in two Latin-American countries (Mexico and Colombia) and of his principal variables for his development of a successful way.

Since result is possible to demonstrate some similarities and differences concerning this knowledge of own content of the physical education across a comparative study of couples that play in the mentioned countries, hereby to contribute to the construction of a new unified knowledge that allows the consolidation of a pedagogic and didactic own model of the physical education and sport teachers, enriching in turn not only his knowledge disciplining but also his Pedagogic General Knowledge and the Didactic Knowledge of the Content (CDC) fundamental factors in the structure of the professional knowledge of physical education teachers.

\footnotetext{
${ }^{1}$ Licenciado en Educación Física de la Universidad Pedagógica Nacional. PhD. en Biomecánica Universidad de Texas (EEUU), Docente de Planta, Universidad Pedagógica Nacional.

${ }^{2}$ Licenciado en Educación Física. Docente Universidad Pedagógica Nacional.
} 
Key words: physical activity, physical education, movement, health, pedagogy, didactics, professional knowledge, pedagogic knowledge of the content, knowledge to discipline or of content.

Fecha de recepción: 30 de Septiembre de 2010

Fecha de aprobación: 2 de Noviembre de 2010

\section{Introducción}

El movimiento es una de las cualidades innatas de los seres humanos de mayor importancia y relevancia, más allá del aspecto biológico y funcional. Es concebido como fuente de conocimiento, comunicación, sentimiento y emociones, salud, esparcimiento, placer, estética, desarrollo filogenético y ontogenético. Y como tal, merece ser revisado dentro del saber pedagógico y más exactamente sobre el conocimiento profesional de los profesores de educación física y deportes.

A través de la historia y de la evolución de los seres humanos, existen diversas disciplinas que han abordado el "estudio del movimiento" desde diferentes puntos de vista científicos y pedagógicos. Dentro de estas disciplinas tenemos:

- Fisiología: procesos biológicos

- Biomecánica y kinesiología: procesos físicos.

- Ergonomía: incremento del rendimiento laboral o deportivo.

- Historia y sociología del deporte: manifestaciones deportivas en la cultura.

- Ciencias médicas: procesos de promoción de la salud, prevención y tratamiento de la enfermedad.

- Educación física: que a partir de la reflexión pedagógica aborda procesos de interacción de diferentes disciplinas científicas como las ciencias biológicas y las ciencias sociales y humanas para solucionar campos de problemas mediante los siguientes objetos de estudio:

\begin{tabular}{|l|l|}
\hline Objeto de estudio & Autor \\
\hline Sujeto físicamente bien educado & NASPE/ICHPER - SD \\
\hline El cuerpo y el juego & Ommo Gruppe \\
\hline El hombre en movimiento & José María Cajigal \\
\hline Educación por el movimiento & Jean Le Boulch \\
\hline Cultura física & Lev Matveiev, Caridad Calderón \\
\hline El movimiento como acción & Kurt Meinel \\
\hline
\end{tabular}

\begin{tabular}{|l|l|}
\hline Cultura del movimiento & Bart J. Crum \\
\hline Deporte & $\begin{array}{l}\text { Herbert Haag,Roland Maul,Ommo Gruppe, } \\
\text { Voiger Ritner }\end{array}$ \\
\hline Conducta motriz-acción motriz & Pierre Parlebas \\
\hline Experiencia corporal & Jean Barreau y Jean Morne \\
\hline
\end{tabular}

Los saberes mencionados anteriormente contribuyen a que los profesionales de la educación física aborden estructural y epistemológicamente el movimiento humano como contenido para mejorar su enseñanza en los espacios e instalaciones destinados a sus prácticas pedagógicas. Esto se debe a que el conocimiento del profesor está compuesto de un saber teórico y un saber práctico, siendo este último un "conjunto de conocimientos complejos, orientados a la practicidad que los profesores utilizan activamente para conformar y dirigir el trabajo de la enseñanza" (Elbaz,1983).

Desde esta perspectiva, los profesores no son sujetos pasivos y meramente ejecutores de postulados y teorías educativas elaboradas por expertos, son agentes que alcanzan un cierto grado de autonomía, que emplean en función de su experiencia profesional y de su singular modo de comprender e interpretar el contexto educativo en el que intervienen. En relación con el contenido del conocimiento practico, Elbaz (1983) distingue cinco categorías:

\begin{tabular}{|l|l|}
\hline Categoría & Descripción \\
\hline Conocimiento de sí mismo & $\begin{array}{l}\text { Se refiere a los valores, las actitudes, las } \\
\text { creencias y sentimientos personales del } \\
\text { profesor. }\end{array}$ \\
\hline Conocimiento del medio & $\begin{array}{l}\text { Indica los diversos contextos en los que se } \\
\text { desenvuelve el profesor: aula, escuela, comu- } \\
\text { nidad, etc., y la manera como influyen en la } \\
\text { mentalidad y actividad del docente. }\end{array}$ \\
\hline $\begin{array}{l}\text { Conocimiento de la asig- } \\
\text { natura }\end{array}$ & $\begin{array}{l}\text { Representa el conjunto de saberes teóricos y } \\
\text { los procedimientos que el profesor emplea en } \\
\text { la enseñanza. }\end{array}$ \\
\hline Conocimiento del currículo & $\begin{array}{l}\text { Se refiere a los diferentes elementos que } \\
\text { incluye el proceso de enseñanza aprendizaje. }\end{array}$ \\
\hline $\begin{array}{l}\text { Conocimiento de la ins- } \\
\text { trucción }\end{array}$ & $\begin{array}{l}\text { Indica el enfoque de enseñanza e incluye las } \\
\text { creencias y teorías sobre el aprendizaje que } \\
\text { ha interiorizado el profesor. }\end{array}$ \\
\hline
\end{tabular}

Este conocimiento práctico, a pesar de que para algunos autores que lo denominan el "conocimiento didáctico del contenido" está más focalizado en las teorías cognitivas de los docentes y máxime cuando 
se utilizan didácticas especificas en la enseñanza de actividades deportivas, recreativas, de ejercitación y de motricidad en general que generan competencias en la condición física, habilidades técnicas, diversión y lúdica, entre otras.

En este sentido y más concretamente, el concepto de actividad física se encuentra en boga en diferentes países avanzados y en proceso de desarrollo, se circunscribe a cualquier movimiento corporal voluntario producido por los músculos esqueléticos que conlleva a un mayor gasto de energía, circunstancia que ha traído debate especialmente a las comunidades académicas iberoamericanas, que bajo ese concepto establecen un sentido y significado mucho más amplio, que también genera discrepancias polisémicas y epistemológicas.

El concepto definido anteriormente haría parte del conocimiento profesional de los profesores de educación física, que andan actualizados en cuanto a los diferentes fenómenos y paradigmas que abarca este saber y disciplina vs. aquellos cuyo conocimiento resulta de un proceso constructivo realizado a través de su práctica y en su relación con diferentes actores de la sociedad.

Para Vasco (1985), las estructuras cognitivas de los sujetos no son constantes sino por el contrario evolucionan; en el problema que se asume en el presente documento, las percepciones, habilidades, conocimientos y diferentes experiencias de los profesores, les permite reflexionar, razonar y establecer relaciones no solamente desde el punto de vista declarativo sino también procedimental y condicional.

Morin (2000), profundiza sobre la envergadura de la temática:

El conocimiento de los problemas claves del mundo, de las informaciones claves concernientes al mundo, por aleatorio y difícil que sea, debe ser tratado so pena de imperfección cognitiva, más aún cuando el contexto actual de cualquier conocimiento político, económico, antropológico, ecológico [...] es el mundo mismo. La era planetaria necesita situar todo en el contexto y en la complejidad planetaria. El conocimiento del mundo, en tanto que mundo, se vuelve una necesidad intelectual y vital al mismo tiempo. Es el problema universal para todo ciudadano del nuevo milenio: ¿cómo lograr el acceso a la información sobre el mundo y cómo lograr la posibilidad de articularla y organizarla?, ¿cómo percibir y concebir el contexto, lo global (la relación todo-partes), lo multidimensional, lo complejo? Para articular y organizar los conocimientos y así reconocer y conocer los problemas del mundo, es necesaria una reforma de pensamiento. Ahora bien, esta reforma es paradigmática y no programática: es la pregunta fundamental para la educación ya que tiene que ver con nuestra aptitud para organizar el conocimiento. A este problema universal está enfrentada la educación del futuro porque hay una inadecuación cada vez más amplia, profunda y grave por un lado entre nuestros saberes desunidos, divididos y compartimentados; y por el otro, realidades o problemas cada vez más polidisciplinarios, transversales, multidimensionales, transnacionales, globales y planetarios.

A partir de Christensen (1996), quien reporta que el conocimiento base para la enseñanza promulgado por Shulman, es uno de los aspectos más citados en la formación de profesores luego de la revisión de diversos programas acreditados. Shulman (1987), establece siete categorías, que se presentan a continuación:

1. Conocimiento del contenido, es decir, acerca de la disciplina que se va a enseñar.

2. Conocimiento pedagógico general, describe los métodos de enseñanza, pertinentes para todos los sujetos y situaciones.

3. Conocimiento didáctico del contenido (CDC), establece como enseñar una materia o un tópico para grupos específicos de estudiantes en un contexto específico.

4. Conocimiento del currículo, ayuda a aplicar contenidos y programas desarrollistas apropiados a cada nivel educativo.

5. Conocimiento del contexto educativo, discierne sobre el impacto de los diferentes contextos sobre la instrucción.

6. Conocimiento de los alumnos y sus características, contribuye a aplicar las teorías del aprendizaje humano a la enseñanza. referenciar, objetivos, propósitos y estructuras del sistema educativo. 


\section{Justificación}

Se hace necesario actualizar el conocimiento profesional del profesor en general y específicamente en este caso el de educación física y deportes en la educación superior, que comprende actuaciones, estilos y decisiones que eligen cuando enseñan (Zamudio, 2003). A su vez, como lo señala Duarte (2009) citando Anastasiou, hay que establecer una relación imperiosa entre los conocimientos científicos y los pedagógicos que pasa por el análisis de los conocimientos ganados por las experiencias vividas en las instituciones de enseñanza y en las aulas, haciéndolos punto de partida y de llegada de la reflexión y del proceso de construcción procesal como profesional docente.

Es pertinente aquí indicar que desde hace varios años Porlán y Rivero (1998) describen el conocimiento del profesor como una expresión un tanto suigéneris en la que se entremezclan teorías provenientes del saber científico, con manifestaciones particulares del mundo experiencial cotidiano, con sus hábitos, rutinas, reflexiones, teorías personales y creencias: no es filosófico o metadisciplinar que sigue con rigor las normas epistemológicas del pensamiento científico, pero tampoco se reduce a una expresión simple del saber empírico de la vida escolar; tiene una epistemología propia de un nivel de complejidad al menos similar al del conocimiento científico, aunque tradicionalmente en la formación del profesor no se valora del mismo modo.

Así que el debate que se plantea en esta investigación para los profesionales de la educación física universitaria confronta lo estructural con lo epistemológico, pues existen áreas de estudio que evidencian múltiples enfoques desde los cuales se estudia el movimiento y que llevan a la adquisición de respuestas desde un sólo interés, limitándolo al saber científico positivista, desde el campo del funcionalismo y a la generación de nuevos avances y descubrimientos. Es decir, a la generación de un conocimiento explicativo, descriptivo, comprobable y medible. De otro lado, el problema epistemológico se centra en la idea que el movimiento obedece a comportamientos, sistemas de ideas, valores y creencias, relaciones sociales y, en general como un proceso de intervención humana en el que existe una sinergia de todos estos elementos trayendo consecuentemente acuerdos entre comunidades académicas, paradigmas y teorías.
En los últimos tiempos se amplía el espectro del movimiento humano lo que conlleva diversas denominaciones de las prácticas corporales. Dentro de esa consideración surge recientemente la concepción de los expertos sobre la actividad física y su evidente papel protagónico en la intervención e incidencia sobre el cuerpo de cada sujeto y su entorno. Esta práctica entiende el movimiento como un patrón de incidencia no sólo exógeno, sino también como el establecimiento de una relación endógena con el sujeto. Se concibe el movimiento como la alteración del medio interno de la persona, acción que consecuentemente genera un gasto metabólico mayor al del funcionamiento normal (basal) de su organismo.

Para una mejor apropiación de la conceptualización de actividad física y su estrecha relación con la salud, que coadyuva a fortalecer el conocimiento de los profesores de educación física, es importante citar documentos elaborados recientemente en el contexto latinoamericano.

En esa dirección D’ Amico y Bolívar (2007) manifiestan que:

El sentirse bien, vivir bien o estar bien, es una condición cultural de coexistencia estrechamente relacionada con la buena salud, ésta, definida por la Organización Mundial de la Salud (oms) como un estado de completo bienestar físico, mental y social, y no solamente como la ausencia de la enfermedad, puesto que, en el organismo saludable se integran varias dimensiones -física, social, emocional, intelectual, espiritual y ocupacional- de la vida de la persona, es decir, las dimensiones posibles de perfilar y alcanzar mediante el desarrollo multilateral del ser humano desde sus primeros años de vida, lo cual -como educadores físicosnos impone recordar la necesidad de establecer, a través de diversos medios, la importancia vital de habituar a la población a realizar actividades físicas como recurso preventivo ante las enfermedades y elemento generador de buena salud.

\section{Marco teórico}

Para objeto de este estudio se abordan dos corrientes conceptuales de gran relevancia en el ámbito internacional que permiten reflexionar acerca de los propósitos definidos para la actividad física permitiendo un acercamiento a la estructuración de un nuevo concepto y el análisis y la comprensión de los 
conocimientos de los profesores de educación física entorno a este tema.

En una primera instancia se analiza el concepto de actividad física definido por Manuel Vicente Pedraz $^{3}$ que ha marcado una pauta en la conceptualización de la actividad física en el viejo continente. Por otro lado se presenta la definición de actividad física estructurada por la C.D.C. ${ }^{4}$ que responde a algunos de los principios de organizaciones como la OMS $^{5}$ y la ops 6 , concepto que ha sido pilar de algunos de los más representativos programas de actividad física en la región latinoamericana y algunos a nivel mundial.

\section{Concepción de la actividad física planteado por Pedráz}

Pedraz (1988), como estudioso dedicado y consagrado de la actividad física, no otorga diferencia alguna a este término con algunos otros similares como el de "educación física", generando toda una corriente, principalmente en España, que nos lleva a la reflexión del componente educativo en las prácticas corporales. Citado por Pedraz, Locke entiende la educación física como una parte vital al lado de la educación intelectual y moral, con una visión más utilitarista que contribuye a la formación del nuevo individuo que es capaz de ser útil a sí mismo y a su comunidad, en la medida que lo prepara y lo fortalece para enfrentarse al medio.

Pedraz, atribuyéndole las mismas características de la actividad física, ubica a la educación física con la educación intelectual y la educación moral como parte del proceso de formación del individuo que no puede darse por separado. Así lo sustentan algunos pensadores de la época de la ilustración en la cual se realizaron algunos de los aportes más significativos al campo de la educación, donde esta trasciende más allá de la formación del ciudadano o la realización del tipo ideal de humanidad, sino que adquiere una condición de utilidad pública. No en vano, Rousseau

${ }^{3}$ Licenciado en Educación Física, profesor de la asignatura de epistemología de la Educación Física en el Instituto Nacional de Educación Física de León, adscrito a la Universidad de León.

${ }^{4}$ Center for Disease Control and Prevention (CDC). Atlanta, GA, USA.

${ }^{5}$ Organización Mundial para la Salud.

${ }^{6}$ Organización Panamericana de la Salud.

Lúdica pedaqóq. Vol 2, No. 15 (ene- dic. 2010) pp. 103 - 115 y Kant, mencionados por el autor, dedican grandes apartes de sus obras más significativas (Emilio y Pedagogía, respectivamente) a la importancia de la educación física al lado de la educación moral e intelectual.

Se plantea la contribución que hace la actividad física al establecimiento de un modelo de sujeto sano, productivo, propositivo, eficaz, y de un alto sentido humano; características de un sujeto que posibilita un desarrollo constante de la sociedad donde la educación desempeña un rol muy importante en la formación integral del individuo incluyendo aspectos como el desarrollo intelectual, moral, físico y motor al mismo nivel sin privilegiar uno por encima del otro en la medida en que son posibilidades y potencialidades del sujeto.

Estos procesos educativos permiten a la actividad física actuar como mediadora para la generación de procesos para la promoción de un desarrollo integral transformando cambios en la conciencia y en los hábitos de las personas que se verán reflejados a corto, mediano y largo plazo, en cambios positivos en los estilos de vida y en sus niveles de bienestar. De igual manera se entiende la actividad física como medio para la socialización de los sujetos, posibilitando espacios de convivencia y de disfrute por el acto motriz en la medida que se satisfacen sus necesidades y trabajan sobre los propios intereses, gustos, capacidades y posibilidades establecidos por los diferentes grupos sociales.

El objeto de estudio en esta concepción de la actividad física se refiere al estudio del cuerpo, el movimiento y el cuerpo en movimiento donde el concepto trasciende más allá de la realidad del hombre de ser y vivir su cuerpo, es decir, se hace una mayor relevancia a la condición humana del sujeto que interviene en la práctica de la actividad física que lleva implícita una reflexión de dicha actividad.

De la misma manera, la actividad física advierte un proceso educativo que permite la comprensión clara de los objetivos propuestos que contribuyen a la formación integral del individuo y que no sólo se remite a la obtención de beneficios y mejoramiento de carácter orgánico. En este proceso intervienen algunas ciencias tradicionalmente ocupadas de la educación como lo son: la historia, la psicología, la sociología, etc. y son complementadas por otras disciplinas que también han entrado a formar parte de 
las llamadas ciencias del movimiento a partir de la educación física como por ejemplo: la biología, la fisiología, la anatomía, etc. (Haag, 1994).

Calderón (2008), considera además que la educación física, procurará además de servir al sistema escolar, descontextualizarse para favorecer a las comunidades urbanas y rurales carentes de espacios y programas para mejorar su salud, su condición física, su autoestima y desarrollo integral, a través de metodologías tendientes a la construcción de un tejido social proactivo y sano que atienda la niñez y la juventud vulnerable a la drogadicción y al maltrato.

\section{Concepción de actividad física según el C.D.C. (Cen- ter for Disease Control and Prevention)}

Según el American College of Sports Medicine ACSM (2000), el término "actividad física" se refiere a una gama amplia de actividades y movimientos que incluyen actividades cotidianas, tales como caminar en forma regular y rítmica, jardinería, tareas domésticas pesadas y baile. El ejercicio también es un tipo de actividad física, y se refiere a movimientos corporales planificados, estructurados y repetitivos, con el propósito de mejorar o mantener uno o más aspectos de la salud física.

Esta y algunas otras definiciones marcan una postura frente a la actividad física como toda aquella práctica corporal que conlleva un gasto energético por encima de los niveles necesarios para la realización de las funciones naturales del cuerpo del individuo. En este sentido encontramos una estrecha relación entre la práctica de actividad física y los procesos de adaptación biológica que directa o indirectamente se suscitan en el sujeto que la lleva a cabo.

Es claro que independiente de la intencionalidad en el momento de practicar la actividad física, el ser humano es un sujeto susceptible a los cambios y adaptaciones generados por las características y las condiciones de la práctica de esta actividad, algunas de ellas de carácter externo entre los cuales tenemos los factores ambientales, sociales, culturales, pero de la misma forma originadas por otras de carácter interno como las biológicas, fisiológicas, morfológicas, psicológicas, etc. Todas ellas encaminadas a un sólo propósito que es la adaptación y supervivencia del ser humano y la superposición de este frente a estas nuevas dinámicas que se presentan.
Estas adaptaciones generan en el organismo del ser humano una serie de cambios funcionales y estructurales que apuntan al mejoramiento del desempeño físico del sujeto trayéndole grandes beneficios sobre su estado de salud, no sólo a nivel de prevención sino que desempeña un papel relevante en la atención de la enfermedad, no obstante que contribuye en la aceleración de los tratamientos y disminuye los tiempos de recuperación.

Correa J. (2000), resalta que:

Darwin manifestó un interés por las adaptaciones que realizan los animales para lograr vivir en su medio, sus teorías han correlacionado tres conceptos explicando el éxito de la supervivencia; como primer concepto, se tiene el de la adaptación biológica, el cual enuncia que la adaptación es el "estado de encontrarse ajustado al medio" como una característica particular del ser vivo; el segundo concepto tiene que ver con la adaptación fisiológica y fenotípica, la cual se relaciona con los cambios que se generan en el individuo frente a una situación en particular, y por último el concepto evolutivo o de adaptación genotípica que, el cual considera la adaptación como un proceso que ocurre dentro de una población durante el curso de muchas generaciones.

Por esta razón el entrenamiento o la práctica constante y regular de la actividad física es un mecanismo por el cual se generan las adaptaciones fisiológicas, biológicas y psicológicas, de aquí que este proceso debes ser considerado de forma individual ya que cada persona obedece a procesos adaptativos heterogéneos.

Desde el punto de vista positivista, la actividad física se establece en una relación directa con el movimiento, no obstante en esta tendencia todo movimiento implica una actividad física que requiere un gasto energético y metabólico y que conlleva unas implicaciones de carácter fisiológico que genera algunas adaptaciones positivas o negativas sobre el organismo, es decir, la actividad física como un acto de permanente estado de movimiento.

Con el ánimo de hacer claridad en los conceptos y evitar asociaciones erradas y equívocas, es necesario dentro de esta corriente positivista diferenciar rotundamente los conceptos de "actividad física" la cual se encuentra en estrecha relación con el acto energético y metabólico; y el concepto de "ejercicio 
físico" el cual denota un fin a través del cambio que se produce en este gasto energético. Este segundo concepto es genérico netamente a la acción de ejercitarse o al acto de desarrollar estrategias para lograr un objetivo a través de una planificación y una estructuración en aras de alcanzar un mejoramiento en el desempeño el cual no siempre se encuentra ligado al estado de salud de los sujetos pero del cual, indiscutiblemente, se obtiene una ganancia y que es orientado por principios axiomáticos como los son:

\section{- El organismo}

- Carga de tensión

- Intensidad

- Duración

- Adaptación biológica
- Relación fatiga-recuperación

- Estímulos de carga de diferentes niveles

En este sentido se ha tornado conveniente la estructuración de las políticas, planes, programas y proyectos en el ámbito nacional como internacional abordando una noción generalizada la de actividad física como estandarte y como elemento protector de la salud.

La actividad física de acuerdo con Hebbelinck (1981) es una adaptación por parte del hombre de todas sus necesidades desde el punto de vista biológico, psicológico y social en donde el individuo se integra con el medio ambiente y marca como principal punto de divergencia con el ejercicio físico la "Intencionalidad".

\section{Cuadro comparativo de los conceptos}

Los autores presentan en el siguiente cuadro una aproximación de las dos concepciones mencionadas

\begin{tabular}{|c|c|c|}
\hline Corrientes conceptuales & \multirow{2}{*}{ Concepto de A.F. (Pedraz) } & \multirow{2}{*}{$\begin{array}{l}\text { Concepto de A.F. } \\
\text { C.D.C. }\end{array}$} \\
\hline Aspectos generales & & \\
\hline Objetivo & $\begin{array}{l}\text { La A.F. como un componente muy importante en el de- } \\
\text { sarrollo integral del sujeto, paralelamente al desarrollo } \\
\text { intelectual y al desarrollo moral, sin privilegiar uno por } \\
\text { encima del otro. }\end{array}$ & $\begin{array}{l}\text { La A.F. como medio para incidir beneficiosamente en el } \\
\text { estado de salud de los sujetos y que se encuentra encami- } \\
\text { nado a la prevención de enfermedades crónicas no trans- } \\
\text { misibles (ECNT) y a la promoción de la salud. }\end{array}$ \\
\hline Objeto de estudio & $\begin{array}{l}\text { El cuerpo, el movimiento y el cuerpo en movimiento y la } \\
\text { incidencia de este en el entorno como parte esencial en el } \\
\text { proceso educativo del sujeto. }\end{array}$ & $\begin{array}{l}\text { El cuerpo funcional del individuo, el cuerpo como una } \\
\text { maquina y el mejoramiento en su "performance" (ren- } \\
\text { dimiento). }\end{array}$ \\
\hline Metodología & $\begin{array}{l}\text { Es un proceso individual de reflexión y compresión que } \\
\text { realiza el sujeto entorno las diferentes posibilidades de } \\
\text { movimiento como medio de incidencia en el mundo. In- } \\
\text { terviene en todo el proceso de crecimiento y desarrollo } \\
\text { del sujeto y prevalece a lo largo de su vida. }\end{array}$ & $\begin{array}{l}\text { Generalmente son tareas dirigidas que se encuentran } \\
\text { encaminadas a la intervención de órganos y sistemas } \\
\text { funcionales especializados con miras a la obtención de } \\
\text { beneficios sobre el estado de salud del individuo (cardio- } \\
\text { respiratorio, óseo-muscular, etc.). }\end{array}$ \\
\hline Ciencias o disciplinas que intervienen & $\begin{array}{l}\text { Pedagogía, didáctica, psicología, sociología, antropología, } \\
\text { historia, biología, fisiología, biomecánica, entre otras. }\end{array}$ & $\begin{array}{l}\text { Ciencias médicas, biología, fisiología, kinesiología, bio- } \\
\text { mecánica, antropometría, ergonomía, fisioterapia, entre } \\
\text { otras. }\end{array}$ \\
\hline Tipos de sujetos que participan & $\begin{array}{l}\text { Todo tipo de personas ya que hace parte del proceso evo- } \\
\text { lutivo del ser humano y de su desarrollo filogenético y } \\
\text { ontogenético. }\end{array}$ & $\begin{array}{l}\text { Todo tipo de personas o grupos haciendo una discrimi- } \\
\text { nación de sus necesidades y clasificándolos en grupos } \\
\text { poblacionales específicos. }\end{array}$ \\
\hline ¿Quién ejecuta? & $\begin{array}{c}\text { Generalmente el individuo es el encargado de su propio } \\
\text { trabajo dosificación de acuerdo a la integración en sus } \\
\text { hábitos cotidianos. }\end{array}$ & $\begin{array}{l}\text { El individuo, previo diagnóstico y prescripción por par- } \\
\text { te del personal especializado o del área de la medicina } \\
\text { o afines, con el propósito de afectar el estado de salud } \\
\text { del paciente. }\end{array}$ \\
\hline Definición conceptual & $\begin{array}{l}\text { Se entiende por A.F. todas aquellas prácticas corporales } \\
\text { que están mediadas por el movimiento con todas aque- } \\
\text { llas categorías que se subyacen como: ejercicio, deporte, } \\
\text { práctica motriz, etc. "La actividad física, el movimiento } \\
\text { es una cualidad sustancial al ser vivo... sobre todo en el } \\
\text { hombre ha cobrado una dimensión a tenor de la utiliza- } \\
\text { ción que más allá de lo puramente biológico y funcional } \\
\text { ha sabido y podido conferirle [...]". }\end{array}$ & $\begin{array}{l}\text { "El término 'actividad física' se refiere a una gama amplia } \\
\text { de actividades y movimientos que incluyen actividades } \\
\text { cotidianas, tales como caminar en forma regular y rítmi- } \\
\text { ca, jardinería, tareas domésticas pesadas y baile. El ejer- } \\
\text { cicio también es un tipo de actividad física, y se refiere } \\
\text { a movimientos corporales planificados, estructurados y } \\
\text { repetitivos, con el propósito de mejorar o mantener uno } \\
\text { o más aspectos de la salud física” }\end{array}$ \\
\hline $\begin{array}{l}\text { Recursos físicos } \\
\text { infraestructura }\end{array}$ & $\begin{array}{l}\text { Todas aquellas que brinden un espacio de bienestar y co- } \\
\text { modidad al sujeto de acuerdo a sus necesidades e intere- } \\
\text { ses. Pueden ser al aire libre o espacios cerrados. }\end{array}$ & $\begin{array}{c}\text { Pueden ser al aire libre o espacios cerrados con imple- } \\
\text { mentos o medios necesarios para la actividad a realizar } \\
\text { de acuerdo a sus necesidades e intereses. }\end{array}$ \\
\hline Ámbitos & $\begin{array}{c}\text { Escolar } \\
\text { Comunitario }\end{array}$ & $\begin{array}{l}\text { Salud } \\
\text { Escolar } \\
\text { Laboral } \\
\text { Comunitario }\end{array}$ \\
\hline
\end{tabular}




\section{Propósito de la investigación}

El propósito de este estudio fue establecer los conocimientos que tienen los profesionales del deporte y la educación física que cursaban estudios posgraduales en Colombia y México, sobre actividad física relacionada con diferentes variables. Dentro del conocimiento de los profesionales es importante contemplar las concepciones entendiéndolas como "presupuestos o esquemas conceptuales alternativos, en relación con la epistemología y axiología que guían las practicas y que pueden relacionarse con la ideas previas que tengan los individuos" (Díaz y Hernández, 2002).

\section{Participantes}

Los sujetos de este estudio fueron 46 profesionales en educación física y deporte que se encuentran actualmente en ejercicio y quienes a su vez eran estudiantes a nivel de postgrado de la Maestría en Actividad Física y Deporte de la Universidad de Pamplona, en el departamento de Norte de Santander (Colombia) y 60 profesionales en su mayoría del deporte inscritos en el Diplomado en Entrenamiento Deportivo del Instituto Tecnológico de Estudios Superiores de Monterrey (México).

\section{Metodología}

Teniendo en cuenta este panorama de la actividad física en algunos países latinoamericanos a través del análisis de sus propuestas más representativas y exitosas, se pueden deducir algunas generalidades y características de los conocimientos que tienen los profesionales del deporte y la educación física en lo referente a la actividad física y a su vez, establecer un comparativo con sus pares que se desempeñan en otros países latinoamericanos; para el caso del presente estudio se utilizó el método cualitativo de grupos focales (Morgan, 1984 y 1988).

De esta manera el estudio evidencia algunas similitudes y diferencias entre estos dos grupos poblacionales y concibe algunas asociaciones y relaciones que se pueden establecer entre los conocimientos de los profesionales mexicanos y colombianos acerca de cada uno de los paradigmas en actividad física que hemos planteado anteriormente. Esta reflexión se encuentra planteada desde ocho aspectos o variables que hacen referencia a los aspectos más re- levantes en la estructuración e implementación de programas de actividad física como lo son: sujeto, objetivo, condiciones, métodos, medios, instalaciones y costos, implementos y productos.

\section{Resultados}

- Sujeto, hace referencia al individuo quien ejecuta la acción y su incidencia en los procesos personales, en el caso de Colombia la actividad física repercute notablemente en un estilo de vida más saludable a partir de está, responde y adapta los sistemas corporales frente al movimiento, asocia la A.F. como parte del movimiento corporal y sus efectos en las dimensiones psicobio-sociales de la persona y su aplicación en el sujeto ya sea en los campos de la recreación, la lúdica y el deporte.

En el caso de los profesionales de México, esta concepción responde a la calidad de vida en el ser humano a través de la actividad física, el fomento de la cultura en el ser humano y desarrollar armónicamente al individuo en la parte física, psicológica y motriz.

- Objetivo, nos muestra el propósito que tienen los sujetos para realizar actividad física, para los profesionales Colombianos los objetivos se encuentran dirigidos hacia la obtención de salud física, mental, social, cultural, placer, variedad de estética, compensación laboral. A partir de diferentes disciplinas o saberes, exportar conocimiento por medio de herramientas investigativas que generan resultados cualitativos y cuantitativos buscando suplir una necesidad; de igual manera algunas de estas disciplinas y saberes apoyan la A.F. (biología, fisiología, medicina deportiva, biomecánica, etc.).

Los profesionales de México encaminan la A.F. hacia la salud y que contribuyan a una mejor calidad de vida, teniendo en cuenta aspectos como: salud, bienestar, salud emocional e integración social. De la misma forma apunta a la creación de una cultura de la actividad física, promover y fomentar la salud y bienestar personal.

- Condiciones, nos indica los requerimientos básicos necesarios para la práctica de actividad física en los diferentes sectores, en el caso del contexto colombiano los profesionales hacen un mayor 
acento en el ámbito de la salud que permita la una buena condición física, la promoción de hábitos de vida saludable, el desarrollo, el mejoramiento de las cualidades físicas, así mismo, el estimular la generación de políticas públicas encaminadas a la promoción y prevención de las enfermedades crónicas no transmisibles.

Para los sujetos profesionales mexicanos no se considera relevante la población etaria. Y que la práctica de la actividad física no exige condiciones especiales en términos de lugar y sujetos, pero consideran un factor muy importante la buena disposición y la actitud positiva de los practicantes en el desarrollo de la actividad física.

- Métodos, se refiere a las estrategias que se pueden implementar para lograr de manera eficaz y eficiente la consecución de los objetivos propuestos por los practicantes de la actividad física. En los profesionales colombianos estos medios deben ir encaminados hacía la promoción de la autonomía, el ritmo personal de aprendizaje, la responsabilidad y la posibilidad de rotación por diferentes tipos de actividades físicas como medio experiencial y vivencial que permita la exploración y el conocimiento de diferentes propuestas; actividades físicas moderadas y la realización de la A.F. regularmente, de forma individual y colectiva, factores que generan tres formas de realizarla y una de mostrarla con la integración del instructor, una individual que permite tener el control de la A.F. y una grupal que permite la integración con otros actores de la actividad física.

Los profesionales mexicanos consideran importantes las estrategias que involucren el ritmo personal y creativo, la planeación y programación variable y la autonomía, esta última permite la independencia y libertad, con un seguimiento a través de un programa general.

- Medios, son los recursos que se utilizan para llegar a la población objetivo, para los profesionales del medio colombiano, están dirigidos hacia la promoción a partir de campañas, actividades no programadas y eventos comunicativos. Igualmente a partir de una planificación estructurada, sistematizada, interdisciplinar y transdiciplinar.
Estos medios deben poseer unas condiciones como espacio ambiental adecuado y permita la acción pedagógica, lúdica y deportiva.

Los profesionales mexicanos consideran que es necesario valerse de un grupo social u organización, tener diversificación de programas y publicidad, asambleas y actividades aplicables a diferentes grupos sociales u organizaciones.

- Instalaciones-costos, los escenarios deportivos, parques naturales, ciclovías, discotecas, gimnasios, cualquier escenario al aire libre o construcción no costosa. Son las principales estructuras e instalaciones consideradas por los profesionales colombianos; de igual forma las instalaciones y costos dependen del programa o proyecto a ejecutar y de los medios usados, pago de instructores, pago de capacitaciones, instalaciones, escenarios y compras de equipos.

Mientras tanto los escenarios públicos y privados, áreas al aire libre multifuncionales, lugares populares públicos que necesariamente no requieran el pago de cuotas o infraestructura sofisticada son las instalaciones pertinentes para los profesionales mexicanos.

- Implementos, hacen referencia a los materiales de apoyo para la implementación de programas y proyectos de A.F. en Colombia, estos son los implementos deportivos comunes y no es necesario una vestimenta especial. En A.F. dirigida es necesario equipos antropométricos, monitores cardiopulmonares, maquinas para desarrollo de fuerza, software de evaluación para la condición física y test para el mismo, acompañada de la infraestructura acorde con las necesidades y equipos de acuerdo a la A.F. requerida.

Mientras tanto en México, los implementos son los propios del entorno, sin equipo especial, donde prima la adaptación de recursos, el material reciclable sin equipamiento o implementos especiales para su realización. En el caso de actividad física dirigida ropa deportiva personal que brinde comodidad al usuario.

- Productos, es el producto de la materialización de los objetivos, en el caso de los profesionales colombianos consideran que estos deben estar relacionados a la incidencia que se pueda lograr en el mejoramiento de calidad de vida, buenos 
hábitos de actividad física, hábitos saludables, cambios en estilo de vida de los usuarios de los programas y proyectos.

Los profesionales mexicanos consideran que las principales incidencias se deben dar en los campos de la salud, la integración social, un modelo de persona saludable tanto física como mentalmente y la creación, fomento y desarrollo de modelos para salud.

\section{Conclusiones}

De acuerdo con estas informaciones se puede inferir que el conocimiento profesional de los profesores colombianos acerca de la actividad física, conceptualmente se encuentran más encaminadas al paradigma funcional y biológico que se establece desde la CDC en el cual se vincula la práctica de la actividad física con los procesos de adaptación biológica que se suscitan en el sujeto convirtiéndose en un medio para el mejoramiento del estado de salud y por ende de su rendimiento.

En el caso de los conocimientos de los profesionales de México, podemos observar una tendencia un poco más marcada hacia los procesos de desarrollo integral del individuo y su integración con los demás miembros de su comunidad (Pedraz, 1988) en donde no sólo la actividad física contribuya al mejoramiento de un estado de salud sino que también contribuya a una mejor calidad de vida a través de diferentes factores como: salud, bienestar, salud emocional e integración social.

Una parte esencial del conocimiento profesional de los profesores de educación física acerca de actividad física es la relación establecida entre este concepto y el de sedentarismo evidenciando profundas asimetrías sociales y cambios relacionados con los hábitos y prácticas cotidianas de la población, asociando la problemática del "sedentarismo" con las relaciones que se establecen en el ámbito personal, familiar, social y comunitario; en los cuales se han generado hábitos repelentes entorno a la actividad física y el deporte, debido probablemente, al enfoque peyorativo que se la ha dado a través de los años.

De la misma manera, como parte del conocimiento profesional hacen una lectura del contexto en el cual se desarrolla la actividad física, como de sus problemáticas y sus avances como parte del conocimiento práctico planteado anteriormente por Elbaz en el marco teórico de este estudio.

Los profesionales de ambos países dan un especial énfasis en los procesos educativos como parte de ese proceso de formación integral incluyendo aspectos como la formación intelectual, moral y física. En donde la A.F. a través del conocimiento práctico de los profesionales se convierte en un medio para la socialización de los sujetos, posibilitando espacios de convivencia y de disfrute por el acto motriz en la medida que se satisfacen sus necesidades y trabajan sobre los propios intereses, gustos, capacidades y posibilidades establecidos por los diferentes grupos sociales.

Después de un análisis de los conocimientos de cada uno de los grupos de profesionales participantes tanto en Colombia como en México, encontramos algunos puntos de encuentro, lo cual nos marca un parámetro de los conocimientos profesionales que deben tener los profesionales latinoamericanos en educación física, deporte y cultura física probablemente debido a las similitudes que existen entre sus contextos y medios de desempeño.

Sujeto: ambas opiniones buscan que el ser humano a partir de la A.F. obtenga una mejor calidad de vida, además de fomentar cultura y cambios desde el desarrollo armónico del individuo y transformar las dimensiones psico-bio-sociales del sujeto.

Objetivo: dentro de estos encontramos que se plasma el mejoramiento de la salud, bienestar e integración social, generar herramientas que permitan efectuar investigación. En ambos contextos se busca a partir de otras disciplinas y saberes generar apoyo en el ámbito de la A.F. y buscan crear cultura en la A.F. a partir de la promoción y fomento del bienestar y salud personal.

Condiciones: los dos países buscan que las condiciones no limiten los procesos de la actividad física y algo muy importante es que una de las condiciones es no tener un limite en la edad para realizar un ejercicio físico.

En términos de actividad física dirigida se busca que tengan condiciones óptimas para sus procesos y que reúnan la generación de políticas públicas y espacios adecuados. De igual forma, Colombia bus- 
ca promover hábitos y estilos saludables, también promover el desarrollo y mejoramiento de las cualidades físicas que conlleva a prevenir enfermedades crónicas no transmisibles.

Métodos: en las dos naciones estos están basados en la autonomía, el propio ritmo personal y promover la A.F. en forma individual o colectiva.

Medios: hacen énfasis en la promoción y divulgación de eventos y campañas que estén relacionadas con la A.F., de igual manera México propone la diversificación de programas por medio de grupos sociales u organizaciones. En los medios más utilizados para Colombia se incluyen la parte pedagógica, lúdica y didáctica deportiva.

Instalaciones-costos: para ambas naciones son necesarios los escenarios deportivos con óptimas condiciones y la utilización de espacios públicos al aire libre y la adecuación de los mismos que no incurran en costos para la realización de la A.F., igualmente la disposición de material adecuado, preciso y oportuno para la realización de las actividades.

Implementos: en Colombia se ve necesario que a partir de la adecuación estos, se construyan nuevos implementos necesarios para el monitoreo y el seguimiento de la actividad física. Entre tanto, México plantea que los implementos pueden ser tomados del entorno y adaptarse a los recursos que se tienen.

Productos: ambas naciones buscan que el producto de la A.F. sea el mejoramiento de la calidad de vida traducidas en personas con hábitos saludables que posean una integración social positivistas y personas sanas físicas y mentalmente.

Como se puede observar el conocimiento profesional que tienen los profesionales en educación física y deportes de estos dos países se encuentran encaminadas hacia una asociación o tendencia con los dos paradigmas que se plantean al inicio, en los cuales la práctica de la actividad física se encuentra ligada a una serie de factores intrapersonales en donde el sujeto es el responsable de su propia práctica, pero también se encuentra estrechamente relacionada con la influencia de algunos factores de los ambientales que apuntan a generar un nuevo patrón de comportamiento como el cambiar de un estilo de vida sedentario a un estilo de vida un poco más activo, estas variaciones en los comportamientos ge- neralmente se encuentran dirigidas hacia el mejoramiento del estado de salud del sujeto en donde cada una de estas etapas necesitan ser reforzadas con el fin de encaminarlas hacia el objetivo y que se reflejarán inmediatamente en un aumento de condición.

\section{Discusión}

El conocimiento profesional de los profesores de educación física y deporte de México y Colombia en cuanto a actividad física se encuentra bien estructurado desde el punto de vista del conocimiento teórico y el conocimiento práctico resultando este último una parte esencial ya que le permite al profesional construir un conocimiento a partir del entendimiento y la comprensión de las formas de aprendizaje de los individuos y la relación con otros factores que median en este proceso de implementación.

Cada una de las categorías propias del conocimiento profesional del profesor, se encuentran presentes en las respuestas suministradas por los profesores de estos dos países, evidenciando un amplio dominio en los aspectos como el "conocimiento disciplinar" o "conocimiento de contenido" (content knowledge), como una de las categorías definidas por Shulman (1987) para la estructuración de un conocimiento base y que presenta dos subcategorías de trascendental importancia como lo son el "conocimiento de contenido común" (common content knowledge, сск) y el "conocimiento de contenido especializado" (specialized content knowledge, scK) (Ball, Thames \& Phelps, 2008).

En este sentido, se establece como el conocimiento acerca de los beneficios de practicar actividad física de forma regular sobre el estado de salud de las personas, se podría considerar como parte del "conocimiento de contenido" que poseen los profesores de educación física colombianos y mexicanos; en un segundo renglón se ubica el realizar actividad física durante 40 minutos, por cinco días a la semana genera adaptaciones funcionales en el organismo de la persona que la realiza, es considerado parte del "conocimiento común" de los profesores de educación física de ambos países. Finalmente, el "conocimiento especializado" de los profesores de educación física es el saber que realizando tres sesiones al día de 10 minutos de trote continuo, con una intensidad moderada-alta, durante cinco días a la semana, trae mejoras en el sistema cardiovascular de la persona que la realiza. 
De la misma manera, se observa que el conocimiento profesional de los profesores de educación física mexicanos y colombianos se ve reflejado en otras categorías como el "conocimiento pedagógico" que da cuenta de las estrategias que utilizan los docentes para transmitir los conocimientos de contenido que tienen respecto de la actividad física y que generalmente se encuentran afiliados a uno o varios modelos pedagógicos, en el caso de este estudio se ve reflejado una tendencia hacia un modelo constructivista. Este conocimiento pedagógico es puede ser una consecuencia del nivel de formación que presentan la mayoría de ellos en áreas a fines al sector educativo como programas de licenciatura en educación física, pedagogía, educación básica con énfasis, entre otras.

Además del "conocimiento didáctico de contenido" que se puede establecer como la relación que existe entre el conocimiento de contenido y el conocimiento pedagógico y el cual lo podríamos relacionar con los estilos de enseñanza que tiene cada uno de los profesores de acuerdo a, como lo define Shulman (1986), "las formas de representar y formular el objeto de estudio de tal manera que este se haga comprensible para los otros", en esta oportunidad dirigidas a actividades recreativas, lúdicas, de adquisición de habilidades, de ejercitación, entre otras.

En esta investigación el "conocimiento didáctico de contenido de la actividad física" que tienen los profesores de educación física, tiene en cuenta el ritmo personal y creativo, la planeación y programación variable, generando un nivel de autonomía que permite independencia y libertad, con un seguimiento a través de un programa general. Igualmente, tiene en cuenta el grado de responsabilidad del profesor a la hora de prescribir la actividad física a realizar de acuerdo con las características individuales de cada sujeto pero a su vez, las características grupales que le permiten la interacción con otros actores.

Todo esto le permite al profesor identificar cuales son lo errores más frecuentes a la hora de la práctica de actividad física, teniendo en cuenta el conocimiento de contenido ( $\mathrm{CK}$ ) que posee, también le permite generar adaptaciones de acuerdo con las necesidades del contexto y de los practicantes y, fi- nalmente, generar estrategias que puedan lograr adherencia por parte de los usuarios a los programas de actividad física que se formulen.

\section{Referencias}

American College of Sports Medicine (ACSM) (2000). Guidelines for Exercise Testing and Prescription, New York, $6^{\text {th }}$ edition, Lippincott, Williams and Wilkins.

American Journal of Preventive Medicine (2002). Theorical Approaches to the Promotion of Physical Activity, Published by Elsevier Science Inc. Am J. Prev Med 2002, 23 (2S): 15 - 25.

Calderón, A. (1997). Estilo de vida y trabajo saludable, ISS.

Calderón, A. (2008). 'Implicaciones epistemológicas en la formación de profesores de Educación Física: mirada desde los alumnos'. En Revista Digital, Vol 13 - № 123, Buenos Aires.

Correa, J. E. (2000). 'Reflexiones conceptúales de la adaptación biológica y su relación con el ejercicio físico'. En Revista Colombiana de Rehabilitación, Volumen № 2 .

D'amico, R. \& Bolivar. R. (2007). La Actividad física en el Desarrollo Humano, Caracas, Universidad Pedagógica Experimental Libertador.

Devis, J. (2000). Actividad física deporte y salud, España, Inde.

Díaz, F. y Hernández, G. (2002). Estrategias docentes para un aprendizaje significativo, una interpretación constructivista, México, Editorial Mac-Graw Hill.

Duarte, C. (2009). Saber docente en la formación y en la actividad del profesor de la educación física de la escuela básica. Documento sin publicar.

Elbaz, (1983).Teacher thinking a study of practical knowledge, London, Corm Helm

Haag, H. (1994). 'State of the art of Sport Pedagogy'. En Sport Sciencie Review, Volume 3, Number 1, 1-10.

Hebbelinck, M. (1981). 'Woman and Sport'. En Medicine and Sport Science, Volume 14.

Morgán, D. \& Spanish, M. (1984). 'Focus groups: a new tool for qualitative research'. En Qualitative Sociology, 3. $253-270$.

Morgán, D. (1988). Focus groups as qualitative research, Newbury Park. CA, Sage.

Morin, E. (2000). Los siete saberes necesarios para la educación del futuro, Bogotá, Imprenta Nacional.

Pedraz, M. (1988). Teoría pedagógica de la actividad física, Madrid, Gymnos S.A.

Porlan, R y Rivero, A (1998). El conocimiento de los profesores. Serie fundamentos № 9. Colección Investigación y Enseñanza, Sevilla, Diada Editora.

Restrepo, E. \& Málaga, H. (2001). Promoción de la salud: como construir vida saludable, Panamericana. 
Sánchez, F. (1996). La actividad física orientada hacia la salud, España, Biblioteca Nueva.

Shulman, L. (1987). 'Those Who Understand: Knowledge Growth in Teaching'. En Educational Researcher (February), 4-14.

Shulman, L. (1987). 'Knowledge and teaching: Foundations of the New Reform'. En Harvard Educational Review, 57(1).

Taylor, S. y Bogdan, R. (1987). Introducción a los métodos cualitativos de investigación, Buenos Aires, Editorial Paidos.

Vasco, C. (1985). 'Práctica reflexión y praxis'. En Revista Colombiana de Educación 19.

Zamudio, F. (2003). 'El Conocimiento Profesional del Profesor de Ciencias Sociales'. En Revista de Teoría y Didáctica de las Ciencias Sociales № 8, Mérida, Venezuela.

Zuluaga, 0. (2003). Educación y pedagogía una diferencia necesaria, Bogotá, Cooperativa Editorial Magisterio. 